us in the front door. But once we are there nothing much is shown. Where are the workers in the field, the graduate students, the post-docs. Where are the arguments. Some experimentalists and theorists do not believe in black holes. Where are they. The Ustinov character is an advance but he is too passive. We need a character who can force the explainer to acknowledge that some of the ideas are poorly understood and insufficiently tested. We should see some actual data instead of simply being told that an experiment satisfies Einstein's theory to $1 \%$. The human substance is missing and the presentation of shocks and thrills wears thin half-way through.

Journalists have to dig harder for their stories. They need to use the same critical sense they reserve for other subjects and apply it to science reporting. A more critical less idolised approach is needed. Einstein recognised this problem himself: "In my view, there is but one way to bring a great scientist to the attention of a larger public; it is to discuss and explain the problems and solutions which have characterised his life work. Otherwise the result is a banal hero-worship based on emotions and not on insight." [Emphasis added.] Science is not more objective and mysterious than any other human social activity. Everyone in science knows this. It is time to start showing it to the public.

Joe Schwartz is a freelance journalist on leave from the City University of New York.

\section{Foundations of relativity}

\section{P. C. W. Davies}

General Relativity from $A$ to $B$. By Robert Geroch. Pp.225. (University of Chicago Press: Chicago and London, 1978.) £8.40; $\$ 11.95$

TEN years ago introductory books on general relativity were a rarity. Since then, advances in the subject and a greater awareness among students that gravity is an intriguing, even bizarre, branch of physics, have stimulated a stream of works appealing to the bottom end of the market. An enormous number of people want an appetite-wetter that will "get them into" gravity without the traditional years of investment in advanced mathematics, and not only so that they can bore dinner guests with virtuoso expositions about black holes. Often more formal treatments do not succeed in communicating the sense of awe and iconoclasm that stems from topics such as total gravitational collapse, event horizons and singularities in spacetime. In addition, mathematically based texts tend to ignore the reader's struggle for sound physical intuition, and depend upon a sort of intellectual osmosis in which feeling for the physics is supposed to slowly and spontaneously filter through the formulae.

Robert Geroch is a man of outstanding international reputation, whose personal contributions to the subject of general relativity are great. $\mathrm{He}$ is also a skilful expositor, and has taken enormous trouble to write a book in which the reader starts out from basics. The book is a product of a lecture course given to non-science under- graduates at the University of Chicago, but it can be read with profit by science specialists who want to acquire a historical and philosophical perspective, to scrutinise the fundamental concepts that underpin the theory of relativity, and to perceive what is a rather specialised branch of physics, with a human face. The text is liberally punctuated by line drawings, some of which might, but should not, frighten the potential reader browsing through the pages.

Part A of the book dwells, perhaps a little too laboriously, on the image of our world as a spacetime threaded by worldlines of matter. To communicate the crucial and fundamental concept of the relativity of motion, much space is devoted to the old and discredited Aristotelian and Galilean concepts of space and time. These sections certainly help to focus the reader's attention on the basic issues at stake, but carry the danger of making Aristotle seem more reasonable than Einstein. There is a sense in which relativity is equally as elegant and natural as the everyday, intuitive view of space and time, but a historical approach to the subject does not always do it justice.

The greater part of the book (Part B) gets to grips with general relativity in its full splendour. Professor Geroch adroitly guides the reader through the geometrical basis for gravity without burdening him with elaborate formalism or needless jargon. Every new concept is strongly rooted in concrete physics. Meter sticks, clocks, light signals, records-people-inhabit the author's models, observing and measuring all sorts of phenomena. Great emphasis is laid on physical meaning and consistency, on asking and answering exactly what is meant by distance, interval, and so forth. It is quite the most elaborate discussion of the funda- mental aspects of relativity that I have encountered in a non-specialist book.

The price paid for this admirable but extended devotion to "getting it straight" is that Einstein's actual theory does not surface until threequarters of the way through the book, and when it does, perhaps inevitably for a book at this level, it is largely in the form of analogy. This leaves very little room for applying the theory to anything exciting, but a mandatory chapter on black holes is included. Readers who have taken the effort to get this far will find that the weird and wonderful properties of these entities can in fact be understood in a straightforward way now that the spacetime geometry concept has been so thoroughly well explained. Professor Geroch explicitly excuses himself from

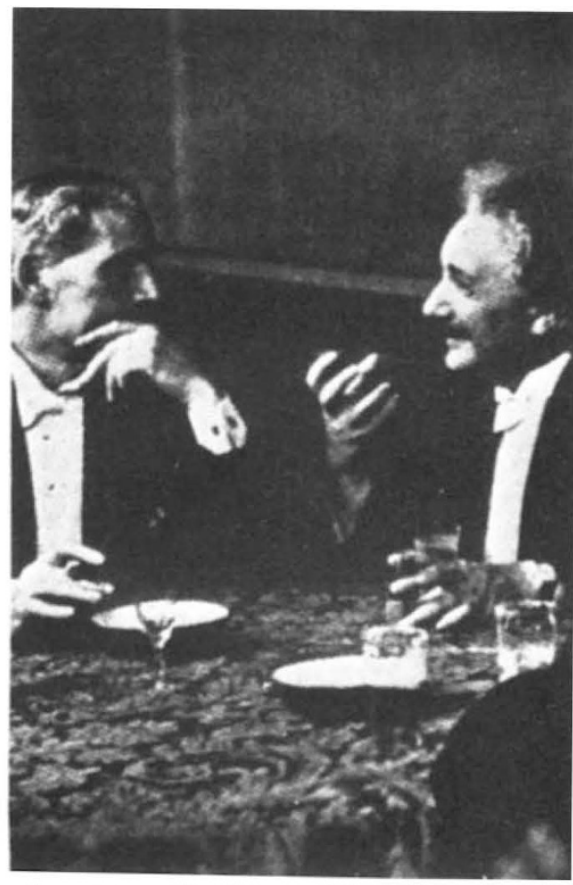

what he calls "gee-whizzes", but still manages to convey the immensely engaging nature of the black holes subject. I was a little disappointed not to see an extensive discussion of singularities, and also the big bang and cosmology, but there is always the possibility of a $C$ to $D$ sequel.

In summary, General Relativity $A$ to $B$ is one of the few books that treats the foundations of the theory of relativity seriously and in elaborate detail, at the non-specialist level. It cannot be read, however, without effort and commitment. The index is rather sparse and the price a shade too high, but otherwise it is a nicely produced book that in spite of its narrow scope illuminates many grey areas of spacetime physics.

P. C. W. Davies is Lecturer in the Department of Mathematics at King's College, University of London, UK. 